\title{
Observation of curative effects of Ex-PRESS and AGV implantation in the treatment of refractory glaucoma
}

\author{
WEN BO, DONGMEI DAI and FENGXIA SUN
}

\begin{abstract}
Department of Ophthalmology, Zaozhuang Ophthalmologic Hospital, Maternity and Child Health Care of Zaozhuang, Zaozhuang, Shandong 277100, P.R. China
\end{abstract}

Received December 14, 2017; Accepted February 16, 2018

DOI: $10.3892 /$ etm.2018.5977

\begin{abstract}
The curative effects of Ex-PRESS implantation and Ahmed glaucoma valve (AGV) implantation in the treatment of refractory glaucoma were compared. A total of 68 patients (76 eyes) with refractory glaucoma treated were randomly divided into Ex-PRESS group and AGV group. Results showed that the intraocular pressure IOP in both Ex-PRESS group and AGV group after operation was significantly decreased compared with that before operation $(\mathrm{P}<0.001)$. At 3,6 and 9 months after operation, IOP in Ex-PRESS group was significantly lower than that in AGV group $(\mathrm{P}<0.05)$. At the end of follow-up, BCVA of patients in both groups after operation was slightly decreased compared with that before operation $(\mathrm{P}>0.05)$. There was no statistically significant difference in BCVA between the two groups of patients $(\mathrm{P}>0.05)$. Moreover, the corneal endothelial cell counts of patients in both groups after operation were obviously decreased compared with those before operation $(\mathrm{P}<0.05)$. The number of anti-glaucoma drugs applied was decreased from $3.53 \pm 0.86$ to $0.55 \pm 0.60$ in Ex-PRESS, and from $3.24 \pm 0.88$ to $0.89 \pm 0.73$ in $\mathrm{AGV}$ group. At the end of follow-up, the number of anti-glaucoma drugs applied in Ex-PRESS group was smaller than that in AGV group $(\mathrm{P}<0.05)$. There was no statistically significant difference in success rate of operation between the two groups at the end of follow-up (log-rank test; P>0.05). Finally, the incidence rates of postoperative complications and surgical intervention rates had no statistically significant differences between the two groups. Ex-PRESS implantation is a safe and effective treatment for refractory glaucoma, characterized by simple operation, small trauma and less pain, which has an equivalent curative effect to AGV implantation; but its control effect on IOP after operation is superior to that of $\mathrm{AGV}$, and
\end{abstract}

Correspondence to: Dr Wen Bo, Department of Ophthalmology, Zaozhuang Ophthalmologic Hospital, Maternity and Child Health Care of Zaozhuang, 6 Xinghua Road, Zaozhuang, Shandong 277100, P.R. China

E-mail: pcwrh247@163.com

Key words: Ex-PRESS implant, Ahmed glaucoma valve, refractory glaucoma, curative effect the number of anti-glaucoma drugs applied after operation is small.

\section{Introduction}

Currently, glaucoma is an irreversible blinding eye disease, ranking first in the world. As of 2020, more than 10 million people around the world have suffered from binocular blindness due to glaucoma. The success rate of filtering operation of refractory glaucoma is only $11-52 \%$, which is much lower than that of conventional glaucoma operation (70-90\%) (1-4).

Since Molteno invented the glaucoma drainage device in 1930, new progress has been made in the treatment of refractory glaucoma (5). On the basis of its drainage principle and structure, some new drainage devices have also emerged; these implantation materials have common characteristics in structure: A flexible silicone tube and an implantation material in the equatorial part of the eyeball, whose common mechanism is that the aqueous humor enters the fiber cavity formed around the implantation material from anterior chamber via silicone tube under the pressure action, and is then absorbed by blood capillaries and lymphatic vessels (6). Ahmed glaucoma valve $(\mathrm{AGV})$ is one of such devices, which significantly improves the success rate of refractory glaucoma operation, and has now become a recognized effective treatment method of refractory glaucoma. However, it has shortcomings of complex operation, low success rate and many postoperative complications, so AGV is always applied cautiously.

Ex-PRESS implant (Ex-PRESS) is a small, stainless steel and splitless glaucoma drainage valve, as well as one of the recently-developed minimally-invasive anti-glaucoma filtering techniques (7). Ex-PRESS is placed under the scleral flap, similar to the traditional trabeculectomy, and it also avoids the removal of sclera and iris required in the trabeculectomy (8). Its theoretical advantages lie in the simplicity and repeatability of operation, and reduction of eye tissue injury. In 2002, upon the approval of Food and Drug Administration (FDA), Ex-PRESS glaucoma drainage device began to be widely used in clinical practice. Ex-PRESS was introduced to China in 2012.

Since Ex-PRESS was widely used in clinical practice, many clinical studies in China and worldwide have compared the treatment effect of Ex-PRESS implantation and trabeculectomy on open-angle glaucoma (9-14), but there is a lack of clinical data on curative effects on secondary glaucoma, 
refractory glaucoma, so further clinical observation is needed. In this study, therefore, the intraocular pressure (IOP) control, success rate of operation, complications, after Ex-PRESS implantation and AGV implantation in the treatment of refractory glaucoma were observed to evaluate the effectiveness and safety of Ex-PRESS implantation in the treatment of refractory glaucoma.

\section{Patients and methods}

General data. A total of 68 patients (76 eyes) with refractory glaucoma treated in Zaozhuang Ophthalmologic Hospital (Zaozhuang, China) from January to December 2016 were enrolled, and they were informed of this study according to the Declaration of Helsinki and signed the informed consent. They were randomly divided into Ex-PRESS group [ $\mathrm{n}=33$ (38 eyes), implanted with Ex-PRESS implant] and AGV group [n=35 (38 eyes), implanted with AGV]. The operation was performed by the same treatment group. In Ex-PRESS group [n=33 (38 eyes)], there were 17 males (17 eyes) and 16 females (21 eyes) aged 19-45 years with an average age of $51.61 \pm 13.76$ years. In AGV group $[n=35$ (38 eyes)], there were 20 males (23 eyes) and 15 females (15 eyes) aged 22-78 years with an average age of $55.27 \pm 13.34$ years. This study was approved by the Ethics Committee of Zaozhuang Ophthalmologic Hospital. Signed written informed consents were obtained from the patients and/or guardians.

Inclusion and exclusion criteria. All patients were aged above 18 years with IOP $>21 \mathrm{mmHg}$. The postoperative follow-up time was less than 9 months, Ex-PRESS group: $11.84 \pm 1.77$ months, AGV group: 12.88 \pm 1.88 months; IOP could not be controlled via systemic and local glaucoma drugs, laser and surgical treatment. Patients with obvious conjunctival scars, adhesions, receiving cyclophotocoagulation, drainage nail or drainage valve implantation were excluded.

IOP measurement. IOP was measured using slit lamp Goldmann tonometer for at least 3 times and the average was taken.

Visual acuity. Best corrected visual acuity (BCVA): Logarithmic visual acuity was converted into LogMAR visual acuity (Table I).

Corneal endothelial cell count. The corneal endothelial cell counts $\left(\mathrm{n} / \mathrm{mm}^{2}\right)$ before operation and at 3,6 and 9 months after operation were obtained using the non-contact corneal endothelial cell counter.

Implantation materials. Ex-PRESS (Alcon Laboratories, Worth, TX, USA) is a new type of aqueous drainage device consisting of a valve-free casing pipe for drainage of aqueous fluid and a protective device preventing the displacement of drainage device. The material is the same stainless steel as the artificial cardiac valve, which has a good biocompatibility with the body. P50 Ex-PRESS with an inner diameter of $50 \mu \mathrm{m}$ was used in the present study.

FP7 AGV is composed of drainage tube and drainage disk. Silicone drainage tube is $0.63 \mathrm{~mm}$ in outer diameter, $0.30 \mathrm{~mm}$
Table I. Conversion table of logarithmic visual acuity and LogMAR.

\begin{tabular}{lccc}
\hline $\begin{array}{l}\text { Logarithmic } \\
\text { visual acuity }\end{array}$ & LogMAR & $\begin{array}{c}\text { Logarithmic } \\
\text { visual acuity }\end{array}$ & LogMAR \\
\hline 0.01 & 2.0 & 0.25 & 0.6 \\
0.02 & 1.7 & 0.3 & 0.5 \\
0.03 & 1.5 & 0.4 & 0.4 \\
0.05 & 1.3 & 0.5 & 0.3 \\
0.1 & 1.0 & 0.6 & 0.2 \\
0.15 & 0.8 & 0.8 & 0.1 \\
0.2 & 0.7 & 1.0 & 0 \\
\hline
\end{tabular}

in inner diameter, and $25 \mathrm{~mm}$ in length; the area of drainage disk is $\sim 184 \mathrm{~mm}^{2}(13 \times 18 \mathrm{~mm})$ and it is oval and made of polypropylene material. A silicone elastomer valve connects the silicone tube and drainage disk. The silicone elastomer valve is a one-way pressure-sensitive valve that can provide resistance to the outflow of aqueous fluid [open pressure of $1.06-1.33 \mathrm{kPa}(8-10 \mathrm{mmHg})]$.

\section{Surgical methods}

Ex-PRESS implantation procedures. Under subconjunctival infiltration anesthesia, the conjunctival flap (diameter of $3 \times 4 \mathrm{~mm}$ and thickness of $1 / 2$ of scleral flap) with the dome as the base was made, the $0.4 \mathrm{mg} / \mathrm{ml}$ mitomycin C (MMC) cotton was placed under the scleral flap and conjunctival flap for $3 \mathrm{~min}$, and then they were washed with $20 \mathrm{ml}$ normal saline. The $25 \mathrm{G}$ needle was punctured into anterior chamber from the scleral flap corneal limbus gray line parallel to the iris surface, part of aqueous fluid was replaced with viscoelastic substance, and the Ex-PRESS drainage device was implanted. The two angle ends and both sides of scleral flap were sutured using 10-0 suture in an adjustable way for a total of 4 needles; the bulbar conjunctiva at the corneal limbus was sutured for 2 needles. Tobradex eye drops and ointment were applied locally after operation.

AGV implantation procedures. Under retrobulbar anesthesia with $1.5 \mathrm{ml} 2 \%$ lidocaine, the conjunctival flap with nasal or supertemporal quadrant as the base was selected; the bulbar conjunctiva and subconjunctival tissues were fully separated and the sclera was exposed. Scleral flap ( $4 \times 5 \mathrm{~mm}, 1 / 2$ thickness) was made using drainage tube into the anterior chamber. The $0.4 \mathrm{mg} / \mathrm{ml} \mathrm{MMC} \mathrm{cotton} \mathrm{was} \mathrm{placed} \mathrm{under} \mathrm{scleral} \mathrm{flap} \mathrm{and}$ conjunctival flap for $3 \mathrm{~min}$, and then they were washed with $20 \mathrm{ml}$ normal saline. Before AGV implantation, the normal saline was injected via drainage tube to ensure the smooth valve. The drainage disk was placed on the sclera between two pieces of rectus muscles across the equatorial part of eyeball. The anterior edge of drainage disk was $\sim 13 \mathrm{~mm}$ away from the corneal limbus. The drainage valve was sutured with 10-0 line in sclera. The no. 7 needle was inserted into the anterior chamber via corneoscleral limbus parallel to the iris. The drainage tube was trimmed to incline $45^{\circ}$ upward into the anterior chamber for 2-3 $\mathrm{mm}$. It was parallel to the iris, but 
Table II. Baseline demographic and clinical characteristics of the patients.

\begin{tabular}{lccc}
\hline & $\begin{array}{c}\text { Ex-PRESS } \\
\text { group } \\
\text { Parameters }\end{array}$ & $\begin{array}{c}\text { AGV } \\
\text { group } \\
(\mathrm{n}-38)\end{array}$ & P-value \\
\hline Sex, $\mathrm{n}(\%)$ & & & 0.192 \\
$\quad$ Male & $17(44.7)$ & $23(60.5)$ & \\
Female & $21(55.3)$ & $15(39.5)$ & \\
Age (years) & $51.61 \pm 13.76$ & $55.27 \pm 13.34$ & 0.246 \\
Follow-up time & $11.84 \pm 1.77$ & $12.08 \pm 1.84$ & 0.57 \\
(months) & & & \\
LogMAR & $0.87 \pm 0.53$ & $1.12 \pm 0.56$ & 0.063 \\
IOP(mmHg) & $36.33 \pm 8.02$ & $34.13 \pm 7.35$ & 0.216 \\
CECC & $2068 \pm 270$ & $1947 \pm 277$ & 0.058 \\
Glaucoma drugs & $3.53 \pm 0.86$ & $3.24 \pm 0.88$ & 0.152 \\
Diagnosis, n (\%) & & & 0.197 \\
NVG & $13(34.2)$ & $15(39.5)$ & \\
Traumatic glaucoma & $9(23.7)$ & $4(10.5)$ & \\
$\quad$ Uveitic glaucoma & $8(21.1)$ & $4(10.5)$ & \\
Filtration surgery & & & \\
failed, n (\%) & & & \\
POAG & $8(21.1)$ & $14(36.8)$ & \\
PKP glaucoma & $0(0)$ & $1(2.6)$ & \\
Previous surgery, $\mathrm{n}(\%)$ & & & 0.463 \\
$\quad$ Trabeculectomy & $23(60.5)$ & $27(71.1)$ & \\
Vitrectomy & $6(15.8)$ & $3(7.9)$ & \\
Keratoplasty & $0(0)$ & $1(2.6)$ & \\
\hline
\end{tabular}

AGV, Ahmed glaucoma valve; IOP, intraocular pressure; CECC, corneal endothelial cell count; NVG, neovascularglaucoma; POAG, primary openangle glaucoma; $\mathrm{PKP}$, penetrating keratoplasty.

did not contact the corneal endothelium and iris. The scleral flap was sutured using 10-0 line for 2 needles and the bulbar conjunctiva at the corneal limbus was sutured using 10-0 line for 2 needles. Tobradex eye drops and ointment were applied locally after operation.

Treatment of complications. In case of complications, drug therapy was preferred based on conditions; if failed, the remedial operative treatment was performed for complications.

Evaluation criteria of operation. Based on literatures in China and worldwide, the evaluation criteria of operation results were summarized as follows: relative success: $5 \mathrm{mmHg}<\mathrm{IOP}$ $<21 \mathrm{mmHg}$, with or without local application of glaucoma drugs; complete success: $5 \mathrm{mmHg}<\mathrm{IOP}<21 \mathrm{mmHg}$, no application of any adjuvant drugs after operation (1). Criteria of operation failure: IOP $>21 \mathrm{mmHg}$ or IOP $<6 \mathrm{mmHg}$; anti-glaucoma surgery was needed again (cyclophotocoagulation or filtering surgery and enucleation of eyeball), the drainage implant needed to be removed or re-implanted, or severe complications (including endophthalmitis, chronic ocular hypotension, malignant glaucoma, retinal detachment and severe choroidal detachment), loss of light sensation or atrophy of eyeball occurred.

Statistical analysis. SPSS 19.0 (SPSS, Inc., Chicago, IL, USA) software was used for statistical treatment. The independent-samples t-test was used for the comparisons of age, preoperative IOP, preoperative and postoperative BCVA, corneal endothelial cell count, postoperative IOP and number of glaucoma drugs applied between the two groups. Chi-square test was used for the comparisons of glaucoma type, sex and postoperative complications between the two groups. Besides, Kaplan-Meier survival curve and log-rank test were adopted for the comparison of success rate of operation between the two groups. A $\mathrm{P}<0.05$ was considered to indicate a statistically significant diference.

\section{Results}

General conditions before operation. There were no significant differences in sex, age, preoperative IOP, preoperative BCVA, corneal endothelial cell count, number of glaucoma drugs applied and history of eye operation between Ex-PRESS group and AGV group $(\mathrm{P}>0.05)$. In Ex-PRESS group, there were 13 eyes with neovascular glaucoma, 9 with traumatic glaucoma, 8 receiving failed glaucoma filtering operation repeatedly, and 8 with secondary glaucoma to inflammation. In AGV group, there were 15 eyes with neovascular glaucoma, 4 with traumatic glaucoma, 14 receiving failed glaucoma filtering operation repeatedly, 4 with secondary glaucoma to inflammation and 1 receiving keratoplasty. There was no significant difference in glaucoma type between the two groups of patients $(\mathrm{P}>0.05)$. The results are shown in Table II.

Comparison of average IOP between the two groups before and after operation. At 9 months after operation, IOP was decreased from $35.6 \pm 9.1$ to $13.7 \pm 5.1 \mathrm{mmHg}$ in Ex-PRESS group, and from $33.9 \pm 8.9$ to $17.0 \pm 6.8 \mathrm{mmHg}$ in AGV group. IOP in both groups after operation was significantly decreased compared with that before operation $(\mathrm{P}<0.05)$. At 3, 6 and 9 months after operation, IOP in Ex-PRESS group was obviously lower than that in AGV group $(\mathrm{P}<0.05)$ (Fig. 1).

Comparisons of BCVA and corneal endothelial cell counts between the two groups before and after operation. In Ex-PRESS group, BCVA was $0.72 \pm 0.49 \log$ MAR and the corneal endothelial cell count was $2068 \pm 270 / \mathrm{mm}^{2}$ before operation; BCVA was $1.09 \pm 0.72 \log \mathrm{MAR}$ and the corneal endothelial cell count was $1809 \pm 423 / \mathrm{mm}^{2}$ at the end of follow-up. In AGV group, BCVA was $0.80 \pm 0.61 \log$ MAR and the corneal endothelial cell count was $1947 \pm 277 / \mathrm{mm}^{2}$ before operation; BCVA was $1.15 \pm 0.90 \log$ MAR and the corneal endothelial cell count was $1775 \pm 569 / \mathrm{mm}^{2}$ at the end of follow-up. BCVA in both groups of patients at the end of follow-up was decreased compared with that before operation, but the differences were not statistically significant $(\mathrm{P}>0.05)$. Besides, the corneal endothelial cell counts in both groups of patients at the end of follow-up were also decreased compared with those before operation, and the differences were statistically significant $(\mathrm{P}<0.05)$. At the end of follow-up, BCVA and corneal endothelial cell count had no statistically significant 


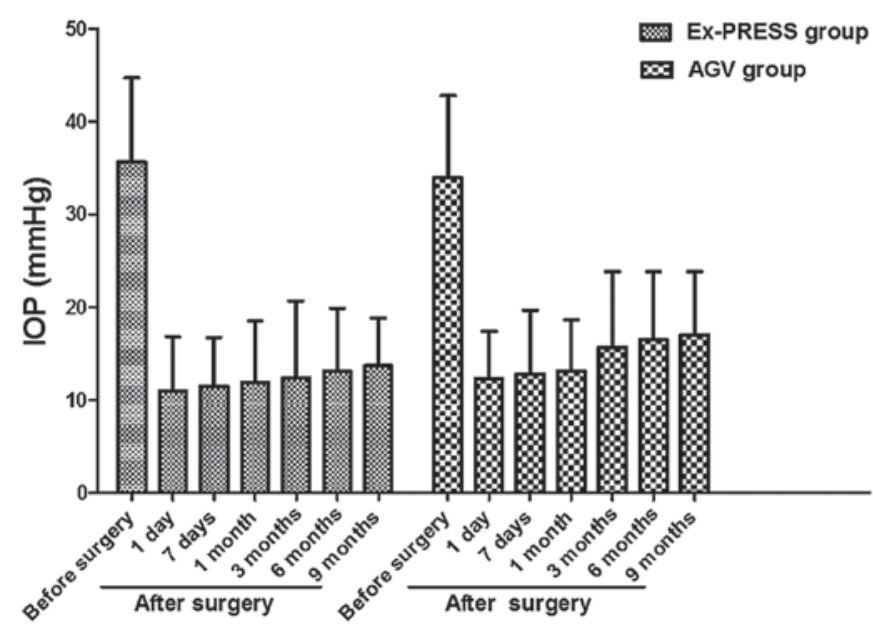

Figure 1. IOP of the study patients in the Ex-PRESS and AGV group before surgery and 1 day, 7 days, 1 month, 3, 6 and 9 months after surgery. IOP of the study patients in the two groups was dramatically decreased after surgery $(\mathrm{P}<0.001)$. IOP of the study patients in the Ex-PRESS group was significantly lower than that of AGV group in 3, 6 and 9 months after surgery $(\mathrm{P}<0.05)$. AGV Ahmed glaucoma valve; IOP, intraocular pressure.
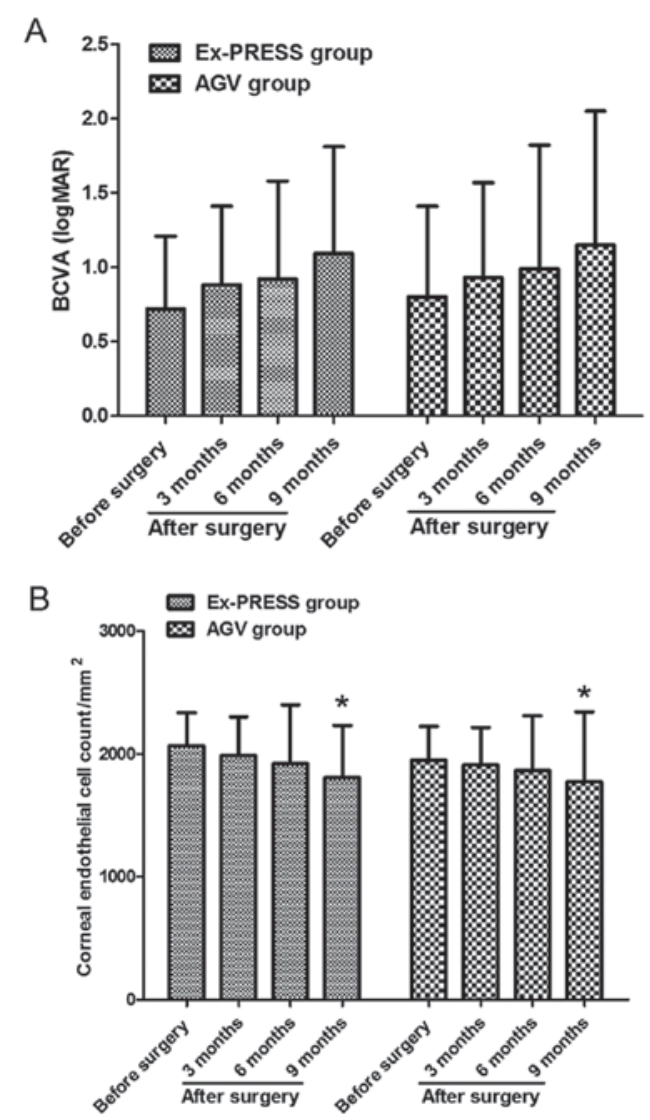

Figure 2. (A) The BCVA and (B) corneal endothelial cell count of the study patients in the Ex-PRESS group and AGV group before surgery and 3, 6 and 9 months after surgery. "Compared with before surgery, $\mathrm{P}<0.05$. BCVA, best corrected visual acuity; AGV, Ahmed glaucoma valve.

differences between the two groups of patients $(\mathrm{P}>0.05)$ (Fig. 2).

Application of anti-glaucoma drugs in the two groups before and after operation. At 9 months after operation, the number
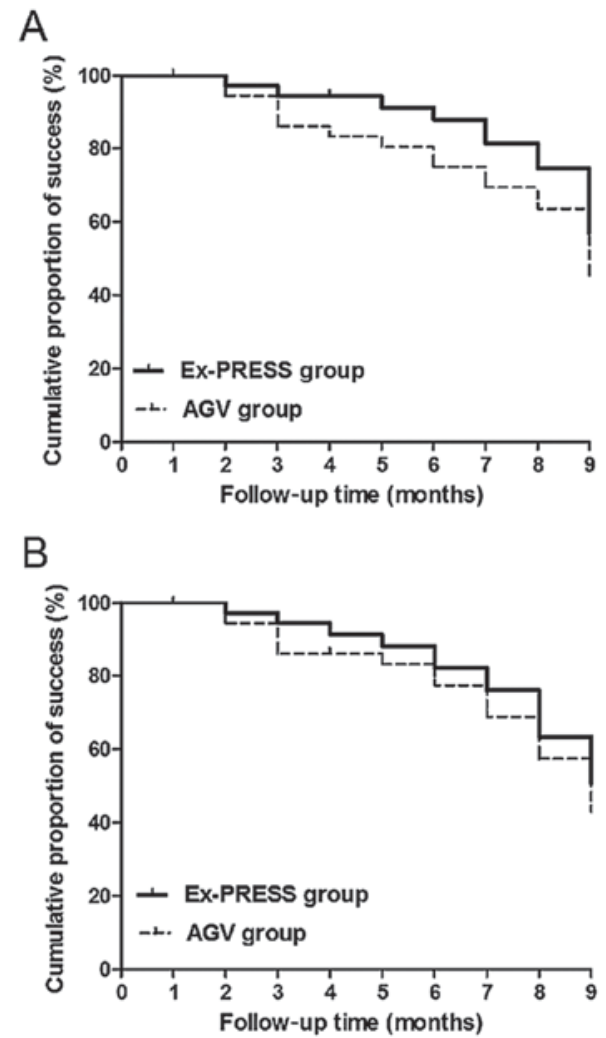

Figure 3. Kaplan-Meier survival curve showed that there was no statistically significant difference in (A) relative success rate and (B) complete success rate of operation between Ex-PRESS group and AGV group at the end of follow-up. AGV, Ahmed glaucoma valve.

Table III. The number of anti-glaucoma drugs used in patients before and after surgery.

\begin{tabular}{lccc}
\hline & \multicolumn{2}{c}{ Groups } & \\
\cline { 2 - 3 } Follow-up time & Ex-PRESS & \multirow{2}{*}{ AGV } & \multirow{2}{*}{ P-value } \\
\hline Before surgery & $3.53 \pm 0.86$ & $3.24 \pm 0.88$ & 0.152 \\
1 month after surgery & $0.18 \pm 0.39$ & $0.26 \pm 0.50$ & 0.448 \\
3 months after surgery & $0.34 \pm 0.63$ & $0.47 \pm 0.65$ & 0.371 \\
6 months after surgery & $0.42 \pm 0.55$ & $0.63 \pm 0.59$ & 0.112 \\
9 months after surgery & $0.55 \pm 0.60$ & $0.89 \pm 0.73$ & 0.029 \\
\hline
\end{tabular}

None of the patients in the two groups used any anti-glaucoma drug in 1 month after surgery. AGV, Ahmed glaucoma valve.

of anti-glaucoma drugs applied was decreased from $3.53 \pm 0.86$ to $0.55 \pm 0.60$ in Ex-PRESS group, and from $3.24 \pm 0.88$ to $0.89 \pm 0.73$ in AGV group; the number of anti-glaucoma drugs applied in Ex-PRESS group was smaller than that in AGV at 9 months after operation ( $\mathrm{P}=0.029$; Table III).

Comparison of success rate of operation. Kaplan-Meier survival curve showed that there was no statistically significant difference in relative success rate of operation between Ex-PRESS and AGV group at the end of follow-up (success rates, 65.8 and 52.6\%, $\mathrm{P}=0.26$, log-rank test) (Fig. 3A). The 
Table IV. Postoperative complications and surgical treatment.

\begin{tabular}{lcr}
\hline & \multicolumn{2}{c}{ Groups } \\
\cline { 2 - 3 } Items & Ex-PRESS n, $(\%)$ & AGV n, $(\%)$ \\
\hline Postoperative complications & & 0.599 \\
Hyphema & $7(18.4)$ & $10(26.3)$ \\
Shallow anterior chamber & $5(13.2)$ & $7(18.5)$ \\
Bleb dysfunction & $8(21.1)$ & $5(21.1)$ \\
Transient intraocular hypertension & $5(13.2)$ & $1(13.2)$ \\
Malignant glaucoma & $0(0)$ & $8(21.1)$ \\
Surgical treatment & & $5(13.2)$ \\
Bleb-plasty and 5-FU subconjunctival injection & $8(21.1)$ & $5(13.2)$ \\
Gonioplasty & $3(7.9)$ & $2(5.3)$ \\
Cyclophotocoagulation & $3(7.9)$ & 0.426 \\
Implant removal & $0(0)$ & \\
\hline
\end{tabular}

AGV, Ahmed glaucoma valve.

complete success rate of operation had no statistically significant difference between the two groups at the end of follow-up (success rates, 57.9 and 47.4\%, $\mathrm{P}=0.48$, log-rank test) (Fig. 3B).

Complications. During and after operation, hyphema occurred in 7 eyes (18.4\%) in Ex-PRESS and 10 eyes (26.3\%) in AGV group, and the blood was absorbed spontaneously within 2 weeks after operation. Five eyes (13.2\%) suffered from transient ocular hypertension in each group within 7 days after operation. Shallow anterior chamber occurred in 5 eyes (13.2\%) in Ex-PRESS and 7 eyes (18.5\%) in AGV group after operation, among which 5 eyes were accompanied by ocular hypotension in each group, and 2 eyes in AGV group suffered from degree III shallow anterior chamber early after operation. Moreover, 8 eyes (21.1\%) in each group had the filtering bleb capsule. Besides, malignant glaucoma occurred in 1 eye in AGV group at 1 month after operation. The incidence rates of postoperative complications had no statistically significant differences between the two groups ( $\mathrm{P}=0.599$; Table IV).

Postoperative intervention. Eight eyes (21.1\%) in each group had the filtering bleb capsule, and the subconjunctival injection of filtering bleb needle combined with 5-fluorouracil was needed. Three patients $(7.9 \%)$ with shallow anterior chamber in each group required anterior chamber angioplasty. Malignant glaucoma occurred in 1 eye in AGV group at 1 month after operation, and anterior chamber angioplasty, vitreous cavity drainage and drug therapy were required. In AGV group, 3 eyes had cilio-choroidal detachment, and treatment was performed, such as atropine for mydriasis, local or systemic application of hormones, binocular bandaging, oral administration of methazolamide and intravenous infusion of mannitol; as a result, 2 eyes returned to normal within 7 days, and drug therapy failed in 1 eye with degree III shallow anterior chamber, and the eye was recovered after suprachoroidal drainage and anterior chamber angioplasty. Transient ocular hypertension in both groups declined to normal after blood-absorption drug therapy and adjustable suture release. Three cases (7.9\%) in
Ex-PRESS and 5 cases (13.2\%) in AGV group finally received cyclophotocoagulation, and the implantation materials were removed from 2 cases $(5.3 \%$ ) due to exposure (Table IV).

\section{Discussion}

In this study, the effect and safety of Ex-PRESS and AGV implantation in the treatment of refractory glaucoma were studied, and it was found that the postoperative IOP in both groups was significantly decreased and controlled well. At 3 months after operation, IOP in Ex-PRESS group was significantly lower than that in AGV group. Moreover, the number of glaucoma drugs applied after operation in both groups was significantly reduced; the number of glaucoma drugs applied at 9 months after operation in Ex-PRESS group was obviously smaller than that in AGV group.

The reason for the better control of IOP in Ex-PRESS group may be due to the inconsistent structure of the two drainage materials. Firstly, the aqueous drainage mechanisms of the two kinds of implantation materials were different. Ex-PRESS drainage device is a valve-free casing tube, so the communication between intraocular and extraocular aqueous fluid has no obstacle; due to the fixed diameter $(50 \mu \mathrm{m})$, the postoperative aqueous effluent is fixed, there is less ocular hypotension and the postoperative IOP is stable (15); AGV silicone elastic valve is a one-way pressure-sensitive valve that can provide the resistance to the outflow of aqueous fluid to prevent the excessive drainage of anterior aqueous fluid before the capsule is formed on the surface of the drainage disk, and it can only open when IOP reaches or exceeds the preset value of $8-10 \mathrm{mmHg}$, and close when IOP is $<8 \mathrm{mmHg}$ to prevent the outflow of aqueous fluid (16). Such a pressure structure results in higher IOP after AGV implantation. Secondly, the drainage capsule and fiber proliferation were different after the two types of implantation. The study of Bae et al (17) showed that the filtering bleb fibrous layer in AGV group was significantly thickened compared with trabeculectomy. Similar to trabeculectomy, the aqueous fluid outflow pathway in Ex-PRESS group comes 
from the sclera, and the intraoperative application of MMC can effectively reduce the fiber proliferation between and around the sclera, so that the filtering bleb fiber proliferation is significantly reduced. As posterior filtration in AGV, AGV was placed under the conjunctival flap and Tenon's capsule to drain the anterior aqueous fluid to the rear of Tenon's capsule; the limited capsule around the drainage valve can significantly reduce the outflow of aqueous fluid. Susanna et al (18) studied and also showed that the application of MMC in AGV implantation does not significantly improve the success rate of operation. Overall, compared with AGV, Ex-PRESS aqueous fluid drainage mechanism and postoperative filtration channel formation mechanism are more conducive to the IOP control.

Many studies have shown that the success rate of Ex-PRESS implantation is similar to that of trabeculectomy, but the postoperative complications are significantly reduced compared with trabeculectomy (7-14,19). The 1-year success rate of Ex-PRESS implantation in the treatment of open-angle glaucoma is more than $80 \%$. As far as we know, there are still few clinical reports on Ex-PRESS implantation in the treatment of refractory glaucoma (20). In this study, after Ex-PRESS and AGV implantation for refractory glaucoma, Kaplan-Meier survival curves showed that there were no statistically significant differences in the relative and complete success rates of operation at the end of follow-up between Ex-PRESS group and AGV group; the success rate of AGV implantation was consistent with that in previous studies $(6,21)$, but that of Ex-PRESS implantation was lower than that in previous studies (9-14). This is mainly because of the different types of glaucoma. This study mainly aimed at the refractory glaucoma. It still can be seen from the previous and this study that Ex-PRESS implantation and AGV implantation are undoubtedly effective treatment methods for refractory glaucoma, compared with the drug and laser therapy, although the success rate of operation is decreased over time. This study showed no statistically significant differences in incidence rates of postoperative complications and surgical intervention rates between the two groups.

In conclusion, the results of this study suggested that Ex-PRESS implantation is a safe and effective treatment method of refractory glaucoma, characterized by simple operation, small trauma and small volume of implantation materials, so it is easier to be popularized and applied clinically. However, this study had some limitations. First of all, Ex-PRESS has been applied in China for a short time, so only a small number of cases were included in this study; secondly, there was a certain bias. In order to minimize the bias, a detailed comparative analysis was performed for the homogeneity of patients' general conditions before data statistics, and patients were followed up for at least 9 months, so that the short-term effect of Ex-PRESS implantation could be described and analyzed more reliably. The long-term curative effect of Ex-PRESS implantation on refractory glaucoma remains to be further demonstrated via further large-sample multi-center randomized controlled clinical research.

\section{Acknowledgements}

Not applicable.

\section{Funding}

No funding was received.

\section{Availability of data and materials}

All data generated or analyzed during this study are included in this published article.

\section{Authors' contributions}

WB designed the study, DD collected data, FS analysed the data, WB and FS prepared the manuscript. All authors read and approved the final manuscript.

\section{Ethics approval and consent to participate}

This study was approved by the Ethics Committee of Zaozhuang Ophthalmologic Hospital (Zaozhuang, China). Signed written informed consents were obtained from the patients and/or guardians.

\section{Consent for publication}

Not applicable.

\section{Competing interests}

The authors declare that they have no competing interests.

\section{References}

1. Ansari E: An update on implants for minimally invasive glaucoma surgery (MIGS). Ophthalmol Ther 6: 233-241, 2017.

2. Bikbov MM and Khusnitdinov II: The results of the use of ahmed valve in refractory glaucoma surgery. J Curr Glaucoma Pract 9: 86-91, 2015

3. Gandham SB, Costa VP, Katz LJ, Wilson RP, Sivalingam A, Belmont $\mathbf{J}$ and Smith M: Aqueous tube-shunt implantation and pars plana vitrectomy in eyes with refractory glaucoma. Am J Ophthalmol 116: 189-195, 1993.

4. Ishida K: Update on results and complications of cyclophotocoa gulation. Curr Opin Ophthalmol 24: 102-110, 2013.

5. Molteno AC: New implant for drainage in glaucoma. Clinical trial. Br J Ophthalmol 53: 606-615, 1969.

6. Taglia DP,Perkins TW, Gangnon R, Heatley GA and Kaufman PL: Comparison of the Ahmed glaucoma valve, the Krupin eye valve with disk, and the double-plate Molteno implant. J Glaucoma 11: 347-353, 2002.

7. Nyska A, Glovinsky Y, Belkin M and Epstein Y: Biocompatibility of the Ex-PRESS miniature glaucoma drainage implant. J Glaucoma 12: 275-280, 2003.

8. Dahan E and Carmichael TR: Implantation of a miniature glaucoma device under a scleral flap. J Glaucoma 14: 98-102, 2005.

9. Moisseiev E, Zunz E, Tzur R, Kurtz S and Shemesh G: Standard trabeculectomy and Ex-PRESS miniature glaucoma shunt: A comparative study and literature review. J Glaucoma 24: 410-416, 2015.

10. de Jong LA: The Ex-PRESS glaucoma shunt versus trabeculectomy in open-angle glaucoma: A prospective randomized study. Adv Ther 26: 336-345, 2009.

11. Good TJ and Kahook MY: Assessment of bleb morphologic features and postoperative outcomes after Ex-PRESS drainage device implantation versus trabeculectomy. Am J Ophthalmol 151: 507-13.e1, 2011.

12. de Jong L, Lafuma A, Aguadé AS and Berdeaux G: Five-year extension of a clinical trial comparing the EX-PRESS glaucoma filtration device and trabeculectomy in primary open-angle glaucoma. Clin Ophthalmol 5: 527-533, 2011. 
13. Dahan E, Ben Simon GJ and Lafuma A: Comparison of trabeculectomy and Ex-PRESS implantation in fellow eyes of the same patient: A prospective, randomised study. Eye (Lond) 26: 703-710, 2012.

14. Seider MI,Rofagha S,Lin SC and Stamper RL: Resident-performed Ex-PRESS shunt implantation versus trabeculectomy. J Glaucoma 21: 469-474, 2012.

15. Salim S: Ex-PRESS glaucoma filtration device-surgical technique and outcomes. Int Ophthalmol Clin 51: 83-94, 2011.

16. Wishart PK, Choudhary A and Wong D: Ahmed glaucoma valves in refractory glaucoma: A 7-year audit. Br J Ophthalmol 94: $1174-1179,2010$.

17. Bae K, Suh W and Kee C: Comparative study of encapsulated blebs following Ahmed glaucoma valve implantation and trabeculectomy with mitomycin-C. Korean J Ophthalmol 26: 265-270, 2012.

18. Susanna R Jr; Latin American Glaucoma Society Investigators: Partial Tenon's capsule resection with adjunctive mitomycin $\mathrm{C}$ in Ahmed glaucoma valve implant surgery. Br J Ophthalmol 87: 994-998, 2003
19. Maris PJ Jr, Ishida K and Netland PA: Comparison of trabeculectomy with Ex-PRESS miniature glaucoma device implanted under scleral flap. J Glaucoma 16: 14-19, 2007.

20. Wamsley S, Moster MR, Rai S, Alvim HS and Fontanarosa J: Results of the use of the Ex-PRESS miniature glaucoma implant in technically challenging, advanced glaucoma cases: A clinical pilot study. Am J Ophthalmol 138: 1049-1051, 2004.

21. Yalvac IS, Eksioglu U, Satana B and Duman S: Long-term results of Ahmed glaucoma valve and Molteno implant in neovascular glaucoma. Eye (Lond) 21: 65-70, 2007.

This work is licensed under a Creative Commons Attribution-NonCommercial-NoDerivatives 4.0 International (CC BY-NC-ND 4.0) License. 